\title{
Some Results Concerning Memomorphic Matrix Valued Functions.
}

\author{
${ }^{1}$ Subhas S. Bhoosnurmath, ${ }^{2}$ K.S.L.N.Prasad \\ ${ }^{I}$ Department of Mathematics, Karnatak University, Dharwad-580003-INDIA \\ ${ }^{2}$ Associate Professor, Department of Mathematics, Karnatak Arts College, Dharwad-580001, INDIA
}

Abstract: In this paper we have extended some basic results of Nevanlinna theory to Matrix valued meromorphic functions.

Key words:Nevanlinna theory,Matrix valued meromorphic functions.

\section{Preliminaries:}

By a meromorphic function we shall always mean a transcendental meromorphic function in the plane. If $\mathrm{f}$ is a meromorphic function, $\mathrm{a} \in \overline{\mathrm{C}}$ and $\mathrm{r}>0$, we use the following notations of frequent use in Nevanlinna theory with their usual meaning: $m(r, a, f)=m\left(r \frac{1}{f-a}\right), n(r, a, f)=n\left(r, \frac{1}{f-a}\right)$,

$\overline{\mathrm{n}}(\mathrm{r}, \mathrm{a}, \mathrm{f}), \mathrm{N}(\mathrm{r}, \mathrm{a}, \mathrm{f}), \overline{\mathrm{N}}(\mathrm{r}, \mathrm{a}, \mathrm{f}), \mathrm{T}(\mathrm{r}, \mathrm{f})$,

$\delta(a, f), \Delta(a, f), \Theta(a, f)$ etc..as in [2]

As usual, if $\mathrm{a}=\infty$, then by a zero of $\mathrm{f}-\mathrm{a}$, we mean a pole of $\mathrm{f}$.

We define a meromorphic matrix valued function as in [1].

By a matrix valued meromorphic function $\mathrm{A}(\mathrm{z})$ we mean a matrix all of whose entries are meromorphic on the whole (finite) complex plane.

A complex number $\mathrm{z}$ is called a pole of $\mathrm{A}(\mathrm{z})$ if it is a pole of one of the entries of $\mathrm{A}(\mathrm{z})$, and $\mathrm{z}$ is called a zero of $\mathrm{A}(\mathrm{z})$ if it is a pole of $[\mathrm{A}(\mathrm{z})]^{-1}$.

For a meromorphic $\mathrm{m} \times \mathrm{m}$ matrix valued function $\mathrm{A}(\mathrm{z})$,

$$
\text { let } \mathrm{m}(\mathrm{r}, \mathrm{A})=\frac{1}{2 \pi} \int_{0}^{2 \pi} \log \left\|\mathrm{A}\left(\mathrm{re}^{\mathrm{i} \theta}\right)\right\| \mathrm{d} \theta
$$

where A has no poles on the circle $|z|=r$.

$$
\begin{aligned}
& \text { Here, }\|\mathrm{A}(\mathrm{z})\|=\operatorname{Max}_{\|\mathrm{x}\|=1}\|\mathrm{~A}(\mathrm{z}) \mathrm{x}\| \\
& \mathrm{x} \in \mathrm{C}^{\mathrm{n}}
\end{aligned}
$$

Set $N(r, A)=\int_{0}^{r} \frac{n(t, A)}{t} d t$

where $n(t, A)$ denotes the number of poles of $A$ in the disk $\{z:|z| \leq t\}$, counting multiplicities.

Let $\mathrm{T}(\mathrm{r}, \mathrm{A})=\mathrm{m}(\mathrm{r}, \mathrm{A})+\mathrm{N}(\mathrm{r}, \mathrm{A})$

Definition : Let $A=\left[a_{i j}\right]_{i, j=1}^{m}$ be a memomorphic matrix valued function of finite order. Let a $\in C$.

$$
\begin{aligned}
& \text { Let } \delta\left(a_{i j}\right)=\delta\left(a, a_{i j}\right)=\lim _{r \rightarrow \infty} \frac{m(r, a)}{T\left(r, a_{i j}\right)}=\varlimsup_{r \rightarrow \infty} \frac{N(r, a)}{T\left(r, a_{i j}\right)} \\
& \text { And } \theta\left(a_{i j}\right)=\theta\left(a, a_{i j}\right)=1-\varlimsup_{r \rightarrow \infty} \frac{N(r, a)}{T\left(r, a_{i j}\right)}
\end{aligned}
$$


Also, Let $\delta(\mathrm{a}, \mathrm{A})=\underset{1 \leq i, j \leq \mathrm{m}}{\operatorname{Max}} \delta\left(\mathrm{a}_{\mathrm{ij}}\right)=\underset{1 \leq \mathrm{i}, \mathrm{j} \leq \mathrm{m}}{\operatorname{Max}} \delta\left(\mathrm{a}, \mathrm{a}_{\mathrm{ij}}\right)$

and

$$
\theta(\mathrm{a}, \mathrm{A})=\operatorname{Max}_{1 \leq \mathrm{i}, \mathrm{j} \leq \mathrm{m}} \theta\left(\mathrm{a}_{\mathrm{ij}}\right)=\underset{1 \leq i, j \leq \mathrm{m}}{\operatorname{Max}} \theta\left(\mathrm{a}, \mathrm{a}_{\mathrm{ij}}\right)
$$

We wish to prove the following results.

Theorem 1 : Let $A=\left[a_{i j}\right]_{i, j=1}^{m}$ be a meromorphic matrix valued functions of finite order. If $\mathrm{a} 1, \mathrm{a} 2, \mathrm{a} 3, \ldots . . \mathrm{a}_{\mathrm{q}}$ are distinct numbers, then $\forall 1 \leq \mathrm{i}, \mathrm{j} \leq \mathrm{m}$.

$$
\sum_{k=1}^{q} m\left(r, a_{k}, a_{i j}\right)+N\left(r, \frac{1}{a_{i j}}\right) \leq T\left(r, a_{i j}^{\prime}\right)+S\left(r, a_{k}, a_{i j}\right)
$$

Where $S\left(r, a_{k}, a_{i j}\right)=S\left(r, a_{i j}\right)=0\left\{T\left(r, a_{i j}\right)\right\} r \rightarrow \infty$ through all values of $a_{i j}$.

Theorem 2 :Let $A=\left[a_{i j}\right]_{i, j=1}^{m}$ be a meromorphic matrix valued function of finite order. Then,

$$
\lim _{r \rightarrow \infty} \frac{T\left(r, A^{\prime}\right)}{T(r, A)} \geq \sum_{a \in C} \theta(a, A)
$$

Theorem 3 Let $A=\left[a_{i j}\right]_{i, j=1}^{m}$ be meromorphoic matrix valued function of finite order.

$$
\text { Then, } \lim _{\mathrm{r} \rightarrow \infty} \frac{\mathrm{T}\left(\mathrm{r}, \mathrm{A}^{\prime}\right)}{\mathrm{T}(\mathrm{r}, \mathrm{A})} \leq 2-\theta(\infty, \mathrm{A})
$$

\section{Proof of theorem 1:}

Without loss of generality, let us assume that for $\mathrm{q} \geq 2$,

$$
\mathrm{F}(\mathrm{z})=\sum_{\mathrm{k}=1}^{\mathrm{q}} \frac{1}{\mathrm{a}_{\mathrm{ij}}-\mathrm{a}_{\mathrm{k}}}, 1 \leq \mathrm{i}, \mathrm{j} \leq \mathrm{m} \text {. }
$$

Then by a known result [Hayman, p.33], we have

$$
\mathrm{M}(\mathrm{r}, \mathrm{F}) \geq \sum_{\mathrm{k}=1}^{\mathrm{q}} \mathrm{m}\left(\mathrm{r}, \mathrm{a}_{\mathrm{k},} \mathrm{a}_{\mathrm{ij}}\right)-\mathrm{q} \log +\frac{3 \mathrm{q}}{\delta}-\log 2, \text { where } \delta=\min _{\mathrm{k}_{1} \neq \mathrm{k}_{2}}\left|\mathrm{a}_{\mathrm{k}_{1}}-\mathrm{a}_{\mathrm{k}_{2}}\right|
$$

Therefore, $\quad \sum_{\mathrm{k}=1}^{\mathrm{q}} \mathrm{m}\left(\mathrm{r}, \mathrm{a}_{\mathrm{k},}, \mathrm{a}_{\mathrm{ij}}\right) \leq \mathrm{m}(\mathrm{r}, \mathrm{F})+\mathrm{O}(1)$

$$
\begin{aligned}
& =m\left(r, \frac{1}{a_{i j}^{\prime}} \cdot F \cdot a_{i j}^{\prime}\right)+O(1) \\
& \leq m\left(r, \frac{1}{a_{i j}^{\prime}}\right)+m\left(r, F \cdot a_{i j}^{\prime}\right)+O(1) \\
& \leq m\left(r, \frac{1}{a_{i j}^{\prime}}\right)+m\left(r, \sum_{k=1}^{q} \frac{a_{i j}^{\prime}}{a_{i j} a_{k}}\right)+O(1) \\
& \leq m\left(r, \frac{1}{a_{i j}^{\prime}}\right)+\sum_{k=1}^{q} m\left(r, \frac{a_{i j}^{\prime}}{a_{i j}-a_{k}}\right)+O(1) \\
& =m\left(r, \frac{1}{a_{i j}^{\prime}}\right)+S\left(r, a_{i j}\right)
\end{aligned}
$$


Thus, $\sum_{\mathrm{k}=1}^{\mathrm{q}} \mathrm{m}\left(\mathrm{r}, \mathrm{a}_{\mathrm{k},} \mathrm{a}_{\mathrm{ij}}\right)+\mathrm{N}\left(\mathrm{r}, \frac{1}{\mathrm{a}_{\mathrm{ij}}^{\prime}}\right) \leq \mathrm{T}\left(\mathrm{r}, \frac{1}{\mathrm{a}_{\mathrm{ij}}^{\prime}}\right)+\mathrm{S}\left(\mathrm{r}, \mathrm{a}_{\mathrm{ij}}\right)$

$$
\leq \mathrm{T}\left(\mathrm{r}, \frac{1}{\mathrm{a}_{\mathrm{ij}}^{\prime}}\right)+\mathrm{S}\left(\mathrm{r}, \mathrm{a}_{\mathrm{ij}}\right)
$$

Hence, the result.

\section{Proof of theorem 2:}

Let $\left\{a_{i j}\right\}_{i=1}^{\infty}$ be an infinite sequence of distinct elements of $C$ which includes for each a $\in C, \theta\left(a, a_{i j}\right)>0$.

Then, $\quad \sum_{\mathrm{a} \in \mathrm{c}}^{\mathrm{q}} \theta\left(\mathrm{a}_{\mathrm{i}}, \mathrm{a}_{\mathrm{ij}}\right)=\sum_{\mathrm{a} \in \mathrm{c}} \theta\left(\mathrm{a}, \mathrm{a}_{\mathrm{ij}}\right)$

Let $\mathrm{q}$ be a positive integer. Then by previous theorem,

$$
\begin{aligned}
\sum_{\mathrm{k}=1}^{\mathrm{q}} \mathrm{m}\left(\mathrm{r}, \mathrm{a}_{\mathrm{k},} \mathrm{a}_{\mathrm{ij}}\right)+\mathrm{N}\left(\mathrm{r}, \frac{1}{\mathrm{a}_{\mathrm{ij}}}\right) \leq \mathrm{T}\left(\mathrm{r}, \mathrm{a}_{\mathrm{ij}}^{\prime}\right)+\mathrm{S}\left(\mathrm{r}, \mathrm{a}_{\mathrm{k},} \mathrm{a}_{\mathrm{ij}}\right) \\
\text { Adding } \sum_{k=1}^{q} N\left(r, a_{k}, a_{i j}\right) \text { both sides, we get } \\
\sum_{\mathrm{k}=1}^{\mathrm{q}} \mathrm{T}\left(\frac{1}{\mathrm{a}_{\mathrm{ij}}-\mathrm{a}_{\mathrm{k}}}\right) \leq \mathrm{T}\left(\mathrm{r}, \mathrm{a}_{\mathrm{ij}}^{\prime}\right)+\sum_{\mathrm{k}=1}^{\mathrm{q}} \mathrm{N}\left(\mathrm{r}, \mathrm{a}_{\mathrm{k}}, \mathrm{a}_{\mathrm{ij}}\right)-\mathrm{N}\left(\mathrm{r}, \frac{1}{\mathrm{a}_{\mathrm{ij}}^{\prime}}\right)+\mathrm{S}\left(\mathrm{r}, \mathrm{a}_{\mathrm{ij}}\right) \\
=\mathrm{T}\left(\mathrm{r}, \mathrm{a}_{\mathrm{ij}}^{\prime}\right)+\sum_{\mathrm{k}=1}^{\mathrm{q}} \mathrm{N}\left(\mathrm{r}, \mathrm{a}_{\mathrm{k}}, \mathrm{a}_{\mathrm{ij}}\right)-\mathrm{N}_{0}\left(\mathrm{r}, \frac{1}{\mathrm{a}_{\mathrm{ij}}^{\prime}}\right)+\mathrm{S}\left(\mathrm{r}, \mathrm{a}_{\mathrm{ij}}\right)
\end{aligned}
$$

where $\mathrm{N}_{0}\left(\mathrm{r}, \frac{1}{\mathrm{a}_{\mathrm{ij}}^{\prime}}\right)$ is formed with the zeros of $\mathrm{a}_{\mathrm{ij}}^{\prime}$ which are not zeros of any of $\mathrm{a}_{\mathrm{ij}}-\mathrm{a}_{\mathrm{k}}$.

Since $\mathrm{N}_{0}\left(\mathrm{r}, \frac{1}{\mathrm{a}_{\mathrm{ij}}^{\prime}}\right) \geq 0$, We have

$$
\sum_{k=1}^{\mathrm{q}} \mathrm{T}\left(\mathrm{r}, \frac{1}{\mathrm{a}_{\mathrm{ij}}-\mathrm{a}_{\mathrm{k}}}\right) \leq \mathrm{T}\left(\mathrm{r}, \mathrm{a}_{\mathrm{ij}}\right)+\sum_{\mathrm{k}=1}^{\mathrm{q}} \overline{\mathrm{N}}\left(\mathrm{r}, \mathrm{a}_{\mathrm{k},}, \mathrm{a}_{\mathrm{ij}}\right)+\mathrm{S}\left(\mathrm{r}, \mathrm{a}_{\mathrm{ij}}\right)
$$

Now, $\mathrm{T}\left(\mathrm{r}, \frac{1}{\mathrm{a}_{\mathrm{ij}}-\mathrm{a}_{\mathrm{k}}}\right) \leq \mathrm{T}\left(\mathrm{r}, \mathrm{a}_{\mathrm{ij}}\right)+\mathrm{O}(\log \mathrm{r})$

Thus $(*)$ takes the form

$$
=\mathrm{T}\left(\mathrm{r}, \mathrm{a}_{\mathrm{ij}}\right)+\mathrm{o}\left\{\mathrm{T}\left(\mathrm{r}, \mathrm{a}_{\mathrm{ij}}\right)\right\} \text { as } \mathrm{r} \rightarrow \infty
$$

$$
q T\left(r, a_{i j}\right)<T\left(r, a_{i j}^{\prime}\right)+\sum_{k=1}^{q} \bar{N}\left(r, a_{k}, a_{i j}\right)+S\left(r, a_{i j}\right)
$$

Hence, $\quad \mathrm{q} \leq \lim _{\mathrm{r} \rightarrow \infty} \frac{\mathrm{T}\left(\mathrm{r}, \mathrm{a}_{\mathrm{ij}}^{\prime}\right)}{\mathrm{T}\left(\mathrm{r}, \mathrm{a}_{\mathrm{ij}}\right)}+\sum_{\mathrm{k}=1}^{\mathrm{q}} \varlimsup_{\mathrm{r} \rightarrow \infty} \frac{\bar{N}\left(r, \mathrm{a}_{\left.\mathrm{k}, \mathrm{a}_{\mathrm{ij}}\right)}\right)}{\mathrm{T}\left(\mathrm{r}, \mathrm{a}_{\mathrm{ij}}\right)}+\varlimsup_{\mathrm{r} \rightarrow \infty} \frac{\mathrm{S}\left(\mathrm{r}, \mathrm{a}_{\mathrm{ij}}\right)}{\mathrm{T}\left(\mathrm{r}, \mathrm{a}_{\mathrm{ij}}\right)}$

Therefore, $\quad \mathrm{q} \leq \lim _{\mathrm{r} \rightarrow \infty} \frac{\mathrm{T}\left(\mathrm{r}, \mathrm{a}_{\mathrm{ij}}^{\prime}\right)}{\mathrm{T}\left(\mathrm{r}, \mathrm{a}_{\mathrm{ij}}\right)}+\sum_{\mathrm{k}=1}^{\mathrm{q}}\left\{1-\theta\left(\mathrm{a}_{\mathrm{k}}, \mathrm{a}_{\mathrm{ij}}\right)\right\}+\varlimsup_{\mathrm{r} \rightarrow \infty} \frac{\mathrm{S}\left(\mathrm{r}, \mathrm{a}_{\mathrm{ij}}\right)}{\mathrm{T}\left(\mathrm{r}, \mathrm{a}_{\mathrm{ij}}\right)}$

Taking maximum over $1 \leq \mathrm{i}, \mathrm{j} \leq \mathrm{m}$, we get

$$
\sum_{\mathrm{k}=1}^{\mathrm{q}} \theta\left(\mathrm{a}_{\mathrm{k}}, \mathrm{A}\right) \leq \lim _{\mathrm{r} \rightarrow \infty} \frac{\mathrm{T}\left(\mathrm{r}, \mathrm{A}^{\prime}\right)}{\mathrm{T}(\mathrm{r}, \mathrm{A})}
$$

Hence the result. 


\section{Proof of theorem 3:}

From [1], We know that $m\left(r, \frac{f^{\prime}}{f}\right)=S(r, f)$

Therefore, $\quad m\left(r, a_{i j}^{\prime}\right) \leq m\left(r, a_{i j}\right)+S\left(r, a_{i j}\right)$

$$
\begin{aligned}
& \text { Also, } N\left(r, a_{i j}^{\prime}\right)=N\left(r, a_{i j}\right)+\bar{N}\left(r, a_{i j}\right) \\
& \text { Hence, } T\left(r, a_{i j}^{\prime}\right)=T\left(r, a_{i j}\right)+\bar{N}\left(r, a_{i j}\right)+S\left(r, a_{i j}\right)
\end{aligned}
$$

Therefore, $\frac{\mathrm{T}\left(\mathrm{r}, \mathrm{a}_{\mathrm{ij}}^{\prime}\right)}{\mathrm{T}\left(\mathrm{r}, \mathrm{a}_{\mathrm{ij}}\right)} \leq 1+\overline{\mathrm{N}}\left(\mathrm{r}, \mathrm{a}_{\mathrm{ij}}\right)+\mathrm{O}(1)$

Therefore, $\lim _{r \rightarrow \infty} \frac{T\left(r, a_{i j}^{\prime}\right)}{T\left(r, a_{i j}\right)} \leq 2-\theta\left(\infty, a_{i j}\right)$

Taking maximum over $1 \leq i, j \leq m$, we get $\varlimsup_{r \rightarrow \infty} \frac{T\left(r, A^{\prime}\right)}{T(r, A)} 2-\theta(\infty, A)$

\section{References}

[1]. C. L. PRATHER and A.C.M. RAN (1987) : "Factorization of a class of mermorphic matrix valued functions", Jl. of Math. Analysis, $127,413-422$

[2]. HAYMAN W. K. (1964) : Meromorphic functions, Oxford Univ. Press, London. 